\title{
Further Properties of Cayley Digraphs and Their Applications to Interconnection Networks ${ }^{\star}$
}

\author{
Wenjun Xiao ${ }^{1}$ and Behrooz Parhami ${ }^{2}$ \\ 1 Dept. of Computer Science, South China University of Technology, \\ Guangzhou, 510641, P.R. China \\ wjxiao@scut.edu.cn \\ 2 Department of Electrical and Computer Engineering, \\ University of California, \\ Santa Barbara, CA 93106-9560, USA \\ parhami@ece.ucsb.edu
}

\begin{abstract}
In this short communication, we extend the known relationships between Cayley digraphs and their subgraphs and coset graphs with respect to subgroups and obtain some general results on homomorphism and distance between them. Intuitively, our results correspond to synthesizing alternative, more economical, interconnection networks by reducing the number of dimensions and/or link density of existing networks via mapping and pruning. We discuss applications of these results to well-known and useful interconnection networks such as hexagonal and honeycomb meshes.
\end{abstract}

\section{Introduction}

The fact that Cayley (di)graphs and coset graphs are excellent models for interconnection networks, studied in connection with parallel processing and distributed computation, is widely acknowledged [1], [2], [4]. Many well-known interconnection networks are Cayley (di)graphs or coset graphs. For example, hypercube, butterfly, and cube-connected cycles networks are Cayley graphs, while de Bruijn and shuffle-exchange networks are coset graphs [4], [11].

Much work on interconnection networks can be categorized as ad hoc design and evaluation. Typically, a new interconnection scheme is suggested and shown to be superior to some previously studied network(s) with respect to one or more performance or complexity attributes. Whereas Cayley (di)graphs have been used to explain and unify interconnection networks with some success, much work remains to be done. As suggested by Heydemann [4], general theorems are lacking for Cayley digraphs and more group theory has to be exploited to find properties of Cayley digraphs. In this paper, we explore the relationships between Cayley (di)graphs and their subgraphs and coset graphs with respect

\footnotetext{
* This work was supported by the Natural Science Foundation of China and Guangdong Province.
} 
to subgroups and obtain general results on homomorphism between them. We provide several applications of these results to well-known interconnection networks such as hexagonal and honeycomb meshes. Our Cayley graph addressing scheme of interconnection networks is a unified and elegant representation for network nodes, which efficiently uses the results of group theory. Clearly, this addressing method is superior to those such as in [6] and [10] in this respect. For example, we prove the formula on the distance of the honeycomb network by means of the method of group theory. We think that our method will have further applications for interconnection networks.

Before proceeding further, we introduce some definitions and notations related to (di)graphs, Cayley (di)graphs in particular, and interconnection networks. For more definitions and basic results on graphs and groups we refer the reader to [3], for instance, and on interconnection networks to [5], [7]. Unless noted otherwise, all graphs in this paper are undirected graphs.

A digraph $\Gamma=(V, E)$ is defined by a set $V$ of vertices and a set $E$ of $\operatorname{arcs}$ or directed edges. The set $E$ is a subset of elements $(u, v)$ of $V \times V$. If the subset $E$ is symmetric, that is, $(u, v) \in E$ implies $(v, u) \in E$, we identify two opposite $\operatorname{arcs}(u, v)$ and $(v, u)$ by the undirected edge $(u, v)$. Because we deal primarily with undirected graphs in this paper, no problem arises from using the same notation $(u, v)$ for a directed arc from $u$ to $v$ or an undirected edge between $u$ and $v$. Let $G$ be a (possibly infinite) group and $S$ a subset of $G$. The subset $S$ is said to be a generating set for $G$, and the elements of $S$ are called generators of $G$, if every element of $G$ can be expressed as a finite product of their powers. We also say that $G$ is generated by $S$. The Cayley digraph of the group $G$ and the subset $S$, denoted by $\operatorname{Cay}(G, S)$, has vertices that are elements of $G$ and arcs that are ordered pairs $(g, g s)$ for $g \in G, s \in S$. If $S$ is a generating set of $G$ then we say that $\operatorname{Cay}(G, S)$ is the Cayley digraph of $G$ generated by $S$. If $1 \notin S$ ( 1 is the identity element of $G$ ) and $S=S^{-1}$, then $\operatorname{Cay}(G, S)$ is a simple graph. Assume that $\Gamma$ and $\Sigma$ are two digraphs. The mapping $\phi$ of $V(\Gamma)$ to $V(\Sigma)$ is a homomorphism from $\Gamma$ to $\Sigma$ if for any $(u, v) \in E(\Gamma)$ we have $(\phi(u), \phi(v)) \in E(\Sigma)$. In particular, if $\phi$ is a bijection such that both $\phi$ and the inverse of $\phi$ are homomorphisms then it is called an isomorphism of $\Gamma$ to $\Sigma$. Let $G$ be a (possible infinite) group and $S$ a subset of $G$. Assume that $K$ is a subgroup of $G$ (denoted as $K \leq G$ ). Let $G / K$ denote the set of the right cosets of $K$ in $G$. The (right) coset graph of $G$ with respect to the subgroup $K$ and subset $S$, denoted by $\operatorname{Cos}(G, K, S)$, is the digraph with the vertex set $G / K$ such that there exists an arc $\left(K g, K g^{\prime}\right)$ if and only if there exists $s \in S$ and $K g s=K g^{\prime}$. The following basic result is easily verified.

Theorem 1. The mapping $\phi: g \rightarrow K g$ is a homomorphism from $C a y(G, S)$ to $\operatorname{Cos}(G, K, S)$ for $g \in G$.

\section{Hexagonal Mesh Networks}

Let $G=Z \times Z$, where $Z$ is the infinite cyclic group of integers, and consider $\Gamma=\operatorname{Cay}(G, S)$ with $S=\{(1,0),(-1,0),(0,1),(0,-1),(1,1),(-1,-1)\}$. It is 
evident that $\Gamma$ is isomorphic to the hexagonal (hex) mesh network [10]. A finite hex mesh is obtained by simply using the same connectivity rules for a finite subset of the nodes located within a regular boundary (often a rectangle or hexagon). In the latter case, wraparound links are sometimes provided to keep the node degree uniformly equal to 6 , leading to a hexagonal torus network. Here, we do not concern ourselves with these variations and deal only with the infinite hex mesh. Let $N=\{(d, d, d) \mid d \in Z\}$. Then, $N$ is a normal subgroup of $Z \times Z \times Z$. Let $H=(Z \times Z \times Z) / N$ and $\Sigma=\operatorname{Cos}(Z \times Z \times Z, N, T)$, where $T=\{(1,0,0),(-1,0,0),(0,1,0),(0,-1,0),(0,0,1),(0,0,-1)\}$. Then, it is clear that $\Gamma$ is isomorphic to the Cayley graph $\operatorname{Cay}(H, N T)$ by Theorem 1 , where $N T=\{N t \mid t \in T\}$ is a subset of the group $H$. Now we are prepared to show the following result.

Proposition 1. The network $\Sigma$, defined above, is isomorphic to the hex mesh network.

\section{Proof. Omitted.}

Proposition 1 has interesting applications to parallel and distributed systems, including in certain problems pertaining to cellular communication networks [6].

Using the Cayley-graph formulation of hex mesh networks, we can easily derive the distance $\operatorname{dis}((a, b),(c, d))$ between the vertices $(a, b)$ and $(c, d)$ in such networks.

Proposition 2. In the hex mesh $\Gamma$, dis $((0,0),(a, b))$ equals $\max (|a|,|b|)$ if a and $b$ have the same sign and $|a|+|b|$ otherwise.

Proof. Omitted.

By symmetry of Cayley graphs, we can easily obtain the distance between any two vertices in the graph $\Gamma$ from Proposition 2, using $\operatorname{dis}((a, b),(c, d))$ $=\operatorname{dis}((0,0),(c-a, d-b))$. We also can obtain the routing algorithm of the graph $\Gamma$ from the proof of Proposition 2 directly.

We now consider the automorphism group $A u t(\Gamma)$ of the graph $\Gamma$. We know that $A u t(\Gamma)$ contains the (left) regular automorphism group of $\Gamma$ which is isomorphic to the group $Z \times Z$; we still denote it as $Z \times Z$. Furthermore, we know that $\operatorname{Aut}(\Gamma)=(Z \times Z)(\operatorname{Aut}(\Gamma))_{(u, v)}$, where $(\operatorname{Aut}(\Gamma))_{(u, v)}$ is the stabilizer (subgroup) of $A u t(\Gamma)$ which fixes the vertex $(u, v)$. we easily prove the following.

Proposition 3. Let $\sigma:(x, y) \rightarrow(x, x-y)$ and $\lambda:(x, y) \rightarrow(x-y, x)$ be mappings from $Z \times Z$ to $Z \times Z$. Then, $($ Aut $(\Gamma))=(Z \times Z)<\sigma, \lambda\rangle$, , where $\sigma^{2}=\lambda^{6}=1$ and $\sigma \lambda \sigma=\lambda^{-1}$.

\section{Honeycomb and Other Networks}

Let $G$ be a (possibly infinite) group and $S$ a subset of $G$ and consider the problem of constructing a group $G$ " and its generating set $S$ " such that $G$ " $=G$ as sets and $S " \subseteq S$, and a homomorphism $\phi: \Gamma " \rightarrow \Gamma$, where $\Gamma=\operatorname{Cay}(G, S)$ and $\Gamma "=\operatorname{Cay}\left(G ", S^{\prime \prime}\right)$. It is easily shown that a number of pruning schemes, in- 
cluding the one studied in [8], are equivalent to the construction above. Pruning of interconnection networks constitutes a way of obtaining variants with lower implementation cost, and greater scalability and packageability [9]. If pruning is done with care, and in a systematic fashion, many of the desirable properties of the original (unpruned) network, including (node, edge) symmetry and regularity, can be maintained while reducing both the node degree and wiring density which influence the network cost.

Example 1. In [8], the authors studied the honeycomb torus network as a pruned $2 D$ torus. They also proved that the honeycomb torus network is a Cayley graph, without explicating its associated group. We fill this gap in the following, while also showing (in the proof of Proposition 4 below) why the parameter $k$ in [8] must be even. Let $G=(\langle c\rangle\langle b\rangle)<a\rangle$ be the group generated by the elements $a, b, c$, satisfying the relations $a^{k}=b^{2}=c^{l / 2}=1$, $b c b=c^{-1}, a b a^{-1}=c^{-1} b, a c a^{-1}=c^{-1}$. Here, $k$ and $l$ are even integers. Thus the group $\langle c\rangle\langle b\rangle=\langle c, b\rangle$ is a semidirect product of $\langle c\rangle$ by $\langle b\rangle$, and $G$ is a semidirect product of $\langle c, b\rangle$ by $\langle a\rangle$. Let $S=\left\{a, a^{-1}, b\right\}$ and $\Delta=\operatorname{Cay}(G, S)$. We may prove that $\Delta$ is isomorphic to the honeycomb torus network in [8] (denoted as $\Sigma$ ).

Proposition 4. The Cayley digraph $\Delta$, defined in Example 1, is isomorphic to the honeycomb torus network $\Sigma$ of reference [8].

Proof. Omitted.

Remark 1. We may consider the infinite honeycomb mesh network as a Cayley graph of a different group. Let $G=(\langle c\rangle\langle b\rangle)\langle a\rangle$, where $\langle c\rangle$ and $\langle a\rangle$ are infinite cyclic groups, and $c, b, a$ satisfy the relations $b^{2}=1, b c b=$ $c^{-1}, a b a^{-1}=c^{-1} b, a c a^{-1}=c^{-1}$. Let $S=\left\{a, a^{-1}, b\right\}$ and $\Delta_{\infty}=\operatorname{Cay}(G, S)$. Then $\Delta_{\infty}$ is isomorphic to the infinite honeycomb mesh network.

Now let $N=<a^{k}><c^{l / 2}>$, where $k$ and $l$ are even integers. We can easily verify that $N$ is a normal subgroup of $G$. Construct the quotient group $G^{\prime}=$ $G / N$ and let $S^{\prime}=\left\{N a, N a^{-1}, N b\right\}$; the graph $\operatorname{Cay}\left(G^{\prime}, S^{\prime}\right)$ is isomorphic to the honeycomb torus network.

Remark 2. An important case in the construction above arises for $G=Z_{k_{1}} \times$ $\ldots \times Z_{k_{n}}(n>1)$, where $k_{1}, \ldots, k_{n}$ are positive integers. In general, $G^{\prime \prime}=N \otimes K$ is a semidirect product of groups $N$ and $K$. If $\phi$ is the identity mapping of $G$ to $G$ " , then for $s " \in S$ " we have $\left(x_{1}, \ldots, x_{n}\right) \otimes s "=\left(x_{1}, \ldots, x_{n}\right)+s$ for some $s \in S$. In particular, if $\left(x_{1}, \ldots, x_{n}\right)$ is the identity element of $G$, we obtain that $s "=s$ for some $s \in S$. Hence $S " \subseteq S$. For instance, for the honeycomb torus network, we have $N=\left\langle c><b>, \bar{K}=\left\langle a>, S=\left\{a, a^{-1}, b, b^{-1}\right\}, S^{\prime \prime}=\left\{a, a^{-1}, b\right\}\right.\right.$.

As an application of the method above, we now consider the problem of finding the distance between two vertices in the honeycomb mesh network $\Delta_{\infty}$. We know that the infinite honeycomb mesh network $\Delta_{\infty}=\operatorname{Cay}(G, S)$, where $G=$ $(<c><b>)<a>, S=\left\{a, a^{-1}, b\right\},\langle c>$ and $\langle a>$ are infinite cyclic groups and $c, b, a$ satisfy the relations $b^{2}=1, b c b=c^{-1}, a b a^{-1}=c^{-1} b, a c a^{-1}=c^{-1}$. 
Thus, any element of $G$ can be expressed as the product $c^{j} b^{l} a^{i}$, where $l$ is 0 or 1 and $j$ and $i$ are integers. We first formulate the distance between vertex 1 (the identity of $G$ ) and vertex $c^{j} b^{l} a^{i}$ in the following theorem.

Theorem 2. For $|i| \leq|2 j+l|$, we have dis $\left(1, c^{j} b^{l} a^{i}\right)=\mid 4 j+l+1 / 2\left[(-1)^{i+l}-\right.$ $\left.(-1)^{l}\right] \mid$; otherwise, $\operatorname{dis}\left(1, c^{j} b^{l} a^{i}\right)=|i|+|2 j+l|$.

Proof. Omitted.

Applying the pruning scheme to the infinite mesh, we obtain the infinite honeycomb mesh. By Remark 1 , it is isomorphic to the Cayley graph $\Delta_{\infty}$. Thus by Theorem 2 we have the following.

Corollary 1. In the infinite honeycomb mesh, the distance between nodes $(x, y)$ and $(u, v)$ is obtained as follows: if $|v-y| \leq|u-x|$, then $\operatorname{dis}((x, y),(u, v))$ equals $\left|2(u-x)+1 / 2\left[(-1)^{u+v}-1\right]\right|$ when $x+y \equiv 0(\bmod 2)$, and $\mid 2(x-u)+$ $1 / 2\left[(-1)^{u+v+1}-1\right] \mid$ otherwise. In the case of $|v-y|>|u-x|$, we have $\operatorname{dis}((x, y)$, $(u, v))=|u-x|+|v-y|$.

Proof. Omitted.

Finally, we embark on determining the automorphism group of the infinite honeycomb mesh network. Let $\sigma$ be the mapping of the set $G$ to itself such that $1 \leftrightarrow 1, a \leftrightarrow a^{-1}, b \leftrightarrow b$, and $\sigma^{2}=1$. This is the reflection to the straight line through two vertices 1 and $b$. Similarly, let $\lambda$ be the mapping of the set $G$ to itself such that $1 \leftrightarrow 1, a \leftrightarrow b, a^{-1} \leftrightarrow a^{-1}$, and $\lambda^{2}=1$. The latter is the reflection to the straight line through two vertices 1 and $a^{-1}$. Then we have $(\sigma \lambda)^{3}=1$. We easily prove $\left(\operatorname{Aut}\left(\Delta_{\infty}\right)\right)_{1}=\langle\sigma, \lambda\rangle$. Thus we have proved the following.

Proposition 5. Let the mappings $\sigma$ and $\lambda$ be defined as above. Then, Aut $\left(\Delta_{\infty}\right)=$ $G<\sigma, \lambda>$.

\section{Conclusion}

In this paper, we have provided a number of general results on homomorphism between Cayley (di)graphs and their subgraphs and coset graphs. We have also demonstrated the applications of these results to some well-known interconnection networks, including hexagonal and honeycomb meshes and related networks. Because of the generality of these theorems, which can be viewed as allowing the synthesis of alternative, more economical, interconnection networks by reducing the number of dimensions and/or link density of existing networks via mapping and pruning, we expect that they will find many more applications.

We are currently investigating the applications of our method to the problems related to routing and average internode distance in certain subgraphs of the infinite honeycomb mesh network. These results, along with potential applications in the following areas will be reported in future:

(1) Load balancing and congestion control

(2) Scheduling and resource allocation

(3) Fault tolerance and graceful degradation 
These constitute important practical problems in the design, evaluation, and efficient operation of parallel and distributed computer systems.

\section{Acknowledgment}

The authors are grateful to the referees for their suggestions.

\section{References}

[1] Akers, S.B., Krishnamurthy, B.: A Group Theoretic Model for Symmetric Interconnection Networks. IEEE Trans. Computers,38(1989)555-566

[2] Annexstein, F., Baumslag, M., Rosenberg, A.L.: Group Action Graphs and Parallel Architectures. SIAM J. Computing,19(1990)544-569

[3] Biggs, N.: Algebraic Graph Theory. Cambridge University Press(1993)

[4] Heydemann, M.: Cayley Graphs and Interconnection Networks. In: Graph Symmetry: Algebraic Methods and Applications. (1997)167-224

[5] Leighton, F.T.: Introduction to Parallel Algorithms and Architectures: Arrays, Trees, Hypercubes. Morgan Kaufmann(1992)

[6] Nocetti, F.G., Stojmenovic, I., Zhang, J.: Addressing and Routing in Hexagonal Networks with Applications for Tracking Mobile Users and Connection Rerouting in Cellular Networks. IEEE Trans. Parallel and Distributed Systems, 13(2002)963971

[7] Parhami,B.: Introduction to Parallel Processing: Algorithms and Architectures, Plenum(1999)

[8] Parhami, B., Kwai, D.M.:A Unified Formulation of Honeycomb and Diamond Networks. IEEE Trans. Parallel and Distributed Systems, 12(2001)74-80

[9] Parhami, B., Kwai, D.M.: Incomplete k-ary n-cube and Its Derivatives. J. Parallel and Distributed Computing, to appear.

[10] Stojmenovic, I.: Honeycomb Networks: Topological Properties and Communication Algorithms. IEEE Trans. Parallel and Distributed Systems, 8(1997)1036-1042

[11] Xiao, W.J., Parhami, B.: Some Mathematical Properties of Cayley Digraphs with Applications to Interconnection Network Design. International J. Computer Mathematics, 82(5)(2005)521-528 